\title{
The Evolution of Zhizhuangzhang Initial Consonants in Zhiyan Cluster of Jin Dialect*
}

\author{
Feng Gao \\ School of Literature \\ Xi'an University \\ Xi'an, China 710065
}

\begin{abstract}
The ancient Zhizhuangzhang initial consonants in Zhiyan cluster of Jin dialect belong to Changxu type. The modern pronunciation pattern of Zhizhaozhang groups in Zhiyan cluster is in line with the dialect of "Zhongyuan Yinyun", which was formed at the latest in the Yuan Dynasty.
\end{abstract}

Keywords-ancient Zhizhuangzhang; modern pronunciation type; formation time; Zhiyan cluster; Jin dialect

\section{INTRODUCTION}

Zhiyan cluster of Jin dialect is located in Yan'an City, Shaanxi Province, including dialects of six counties: Wuqi, Zhidan, Ansai, Yan'an, Yanchang and Ganquan. [1] Zhiyan cluster is in the transitional zone between Jin dialect and official language of central China. It has transitional features in many aspects of the dialect. However, the modern pronunciation of Zhinzhuang groups is completely consistent with the mainstream of Jin dialect in Shaanxi and is very stable.

\section{THE MODERN PRONUNCIATION TYPE OF ZHIZHUANGZHANG INITIAL CONSONANTS GROUPS IN ZHIYAN CLUSTER OF JIN DIALECT}

The division and combination rules and the distribution of the pronunciation of ancient Zhizhuangzhang initial consonants in the six counties of Zhiyan cluster of Jin dialect showed a very strong consistency. The modern pronunciations of the ancient Zhizhuangzhang initial consonants are the combination of the Zhierzhuang opening mouth and the Hongyin word of Jing group (except the Dangjiangshezhuang group, the Jiangshezhi group, the Hanzhishezhang group) is read as ts group, the round-mouth combination of Zhisanzhang and Zhuang group (except for the Zhishezhang group, including the Dangjiangshezhuang group and the Jiangshezhi group) is read as ts group, and the modern pronunciation of Dangkaisanzhuang group and the Jiangkaierzhizhuang group evolved with round-mouth characters. There is no variation in the initial consonant of modern pronunciation of the round-mouth characters.

From the classification of the sounds of the ancient Zhizhuangzhang Jing group, there are usually three types of

*This article is one of the achievements of the National Social Science Fund Major Project "Atlas of Northwest Dialect (No. 16:JJD7400 10)". the modern pronunciation of Zhizhuangzhang Jing group initial consonant in Chinese: concourse type, dichotomy type, and trisection type. Zhiyan cluster dialect includes Zhierzhuang and Zhisanzhang, belonging to the dichotomy type. Xiong Zhenghui (1990) pointed out that in the northern dialect, there are the Jinan type with integration of Zhizhuangzhang and the Nanjing type and Changxu type with Zhi'erzhuang and Zhisanzhang opposite to each other. [2] The relationship between the word and sound of Zhizhuangzhang in Zhiyan cluster is the same as that of Changxu type, so it belongs to the typical Changxu type.

\section{THE DisTRIBUTION SCOPE OF THE TYPICAL CHANGXU TYPE}

The dichotomy type of Zhizhuangzhang is widely distributed in Chinese dialects, extending westward to Qinghai, northward to Xinjiang and Inner Mongolia, southward to Xiang dialect and Gan dialect areas, and eastward to Jiaoliao Peninsula. Changxu type is a subtype of dichotomy type, and the distribution scope is also very large: Qinlong cluster (Xining), Guanzhong cluster, Fenhe cluster, Luoxu cluster (Xuzhou), Zhengcao cluster (Ganyu) and south Xinjiang cluster (Yanqi) in the Central Plains Mandarin, North Xinjiang cluster and Hexi cluster (Minqin) of Lanyin official language, Canghui cluster (Cangzhou) and Baotang cluster (Tianjin) of Beijing official language and Qingzhou cluster (Pingdu) and Denglian cluster (Wendeng) of Jiaoliao official language. [3] For example, Haiyuan dialect and Guyuan dialect of Ningxia, Dunhuang dialect and Minqin dialect of Gansu, Xinjiang Urumqi dialect, Xuzhou dialect, etc. are all typical Chang Xu type. So, what is the specific situation of the distribution of typical Changxu type in the dialects around Zhiyan cluster?

The northern part of Zhiyan cluster is the other dialect areas of Jin dielects in the north of Shaanxi. The modern pronunciation of Zhizhuangzhang is relatively uniform, with the typical Changxu type as the mainstream form. Li Jianxiao (2006) researches the pronunciation of the Zhizhuangzhang initial consonants of 21 Jin dialect areas in the northern Shaanxi (the county in the north of Yan'an), and finds that most of the dialects belong to the typical Changxu type, and only six dialect areas are different. The six dialect areas are located in the Suide Zaolinping, Suide Yihe, Jiaxiankeng Town, Wubao Songjiachuan, Wubao Songshang, 
and Wubao Zhangjiashan. The Dangjiangshe open-mouth group, Jiangkai second group and Zhizhuang round-mouth group are read as ts group, and Gengkai second group is read as ts group. [4] This is different from the pronunciation of other Jin dialects areas in Northern Shaanxi but consistent with the dialects of Shanxi Linxian on the other side of the Yellow River. There are 16 dialect areas in central Shanxi that also incorporate Zhizhanzhang round-mouth into Jing Hongyin Group, including dialects in Linxian, Liulin, and Lishi in western district, and Xiaoyi, Jiexiu, Pingding and Heshun in the central district.

They connect to each other geographically, forming a narrow dialect band. [5] Six dialect areas including Kengzhen in northern Shaanxi and Shanxi Linxian and Liulin are on opposite sides of the river, so the Zhizhuangzhang round-mouth ts group should be the result of the influence of the above Shanxi dialect.

The modern pronunciation type of Zhizhuangzhang in Shanxi dialect in the east of Zhiyan cluster is rich. Wang Hongjun (2007) conducted an in-depth study of the modern pronunciation of ancient Zhizhuangzhang group of 101 dialect areas in Shanxi, and divided them into three categories: I Zhizhuangzhang dichotomy type; II Zhizhuangzhang integration type; III Zhizhuangzhang integration type of Jing four groups. The Zhizhuangzhang dichotomy type I include 57 dialect areas with the largest number. They are widely distributed in various regions of Shanxi. Wang Wen continued to subdivide the 57 dialect areas into 4 types. The difference is mainly the conflation and phonological values of the round-mouth pronunciation. The first one is similar to the division and combination of Zhizhuangzhang initial consonants in Zhiyan cluster, all of which belong to the Changxu type. This type is distributed in 29 dialect areas, of which there are 23 areas belonging to the typical Changxu type: Datong, Tianzhen, Shanyin, Shuoxian, Yanggao, Zuoyun, Youyu, Daixian, Fanzhi, Kelan, Baode, Pianguan, Hequ, Zhongyang, Fenyang, Daning, Yonghe, Puxian, Shilou, Huozhou, Xiangning, Yuanqu and Xiangfen. [5] Most of these 23 dialect areas are located in the northern and western parts of Shanxi Province, adjacent to or bordering the north of Shaanxi (including Zhiyan cluster).

Guanzhong dialects in the south of Zhiyan cluster, including five cities: Xi'an, Tongchuan, Baoji, Weinan and Xianyang, Huangling, Huanglong, Luochuan, Yichuan, Fuxian in Yan'an and some counties and cities in Shangluo and Ankang, totaling more than 50 counties and cities belong to the Guanzhong cluster and Qinlong cluster of Central Plain official language. "The ancient Zhi system open-mouth characters in Guanzhong dialect are generally divided according to Zhierzhuang and Zhisanzhang, which belongs to the Changxu type in the [ts ts] type of the Zhi system official dialect. The pronunciation of the round-mouth characters in ancient Zhi system in the Guanzhong dialect is more complicated: the round-mouth characters in 17 dialects areas such as Huaxian in the eastern part of Guanzhong combine with Jing group while in other areas it don't combine. [6] "The open-mouth word in Zhizhuangzhang three groups of Guanzhong dialect also have the tendency to classify according to this. Consistent with the classification of rhyme books after the Yuan Dynasty, it belongs to the Changxu type. Except Zhishe, Zhi'er and Zhuang group have same sound, read as ts group; Zhisan and Zhang group have the same sound, read as the ts class; just for round-mouth characters, the Zhuangzhang are mixed in reading and the sound value varies from region to region." [7] Therefore, the modern pronunciation type of Zhizhuangzhang in Guanzhong dialect should belong to the variable Changxu type.

The west of Zhiyan cluster is Gansu and Ningxia, and its dialect belongs to Qinlong cluster and Longzhong cluster of the Central Plain official language and Longdong area in Gansu is adjacent to Wuqi and Zhidan. There are relatively few materials related to Gansu dialect. "Gansu Dialect Profile" (1960) mentioned that in the dialect of the Longdong Region, "some ts group words are read as ts groups, including the open-mouth two characters of Guzhizhuangzhang groups (except Jiangshe) and the three open-mouth second characters in the same group: "zhi, liu, and shenshe" [8]. The description is not in detail, so the specific distribution area cannot be found. The Zones of Chinese Dialects in the Four Provinces of Shaanxi, Gansu, Ningxia and Qinghai (1986) lists the classification and distribution of the four characters of "zhu, chu, shu, and ru", in which the sound value of initial consonant in Ji type and Ding type didn't change. Ji type are distributed in 11 places such as Wuzhong and Zhongwei in Yinnan cluster, and Jiayuguan, Jiuquan, Yumen, Jinta, Anxi, Wuwei, Minqin, Dunhuang in Hexi cluster, Gulang, Tianzhu, Yinchuan, Yanchi, six places in Lanzhou cluster including Yuzhong and Minqin, ten places in Qinlong cluster, Pingliang and Guyuan, and ten places in Longzhong cluster in Linxia and Lintao. Ding type are distributed in Qingyang, Huanxian, Huachi, Heshui and Dunhuang in Gansu, (Shaanxi) Longxian, Yan'an, Ganquan, Yanchang, Dingbian, Baihe, Pingli, and Zhen'an. [9][10]

It can be seen from the above that the typical Changxu type is mainly distributed in the northwestern part of Shaanxi, the north and west part of Shanxi, the eastern part of Gansu, and most part of Ningxia, which are roughly connected in geographic position. The Zhiyan cluster is located in the central and western part of the cluster.

\section{THE Formation TIME OF MODERN PRONUNCIATION OF ZHIZHUANGZHANG INITIAL CONSONANTS IN ZHIYAN CLUSTER OF JIN DIALECT}

Zhiyan cluster of Jin dialect is neatly divided into two categories according to the ancient sound conditions of Zhierzhuang and Zhisanzhang in "Zhongyuan Yinyun" and there is no irregular change that some words have been changed while some words have not been changed, indicating the modern pronunciation of Zhizhuangzhang is the original level of dialect, instead of the result of the influence of external dialects. The Zhizhuangzhang of Zhiyan cluster is similar to the dialect I of Shanxi Jin dialect. Wang Hongjun believes that "the dialect I should be the native level of dialects" in the three types of Zhizhuangzhang groups in Shanzi dialect. [5] Our view is consistent with that of Mr. Wang Hongjun. 
Zhizhuangzhang initial consonants in Zhiyan cluster of Jin dialect have three characteristics: Zhierzhuang groups and Zhisanzhang are dichotomy, the pronunciation of Zhikaisanzhuang is same to open-mouth Zhuang group, and Jiangkai Erzhizhuang group and Dangkai Sanzhuang group are read as round-mouth words. Some scholars have studied the formation of these three characteristics.

Luo Changpei (1933), through the study of Dunhuang literature reflecting the northwestern dialect pronunciation of the late Tang and Five Dynasties, believed that the Zhizhongzhuang consonants in the northwest China in Tang and Five Dynasties had been mixed. [11] This conclusion is slightly improper. Shao Rongfen (1963) drew the slightly different conclusion through the investigation of the wrongly written characters and strange articles in Dunhuang folk literature and the comparison of Luo Changpei's Chinese and Tibetan audio materials. He holds that "it can be believed that Zhi and Zhang initial consonants in the third-class rhyme can no longer be distinguished", and we seem to be sure that Zhishechongmu and Changmu are mixed. That is to say, only part of the word in Zhuang group is merged into the Zhi and Zhang group, and the Zhuang group is still independent. As for the performance of the second-class Zhi group, Shao Wen did not discuss it. [12] Wanbo (2009) combined the research of each school and compared the following literature materials: Zhang Zilie's "Zhengzitong" in the late Ming and early Qing dynasties, Zhou Deqing's "Zhongyuan Yinyun" in the Yuan Dynasty, Wu Yue's "Yunbu" in the Southern Song Dynasty, Wu Shaoyi's "The Sound Map for Erya" reflecting the actual voice in the Five Dynasties and the early Song Dynasty, and Dunhuang literature reflecting the northwestern dialect of the Tang and Five Dynasties. Finally, the following conclusions are drawn: the two types of Zhierzhuang and Zhisanzhang groups have been formed in the late Tang Dynasty and the Five Dynasties. After North Song Dynasty and South Song Dynasty, Yuan, Ming and Qing dynasties, they remain in modern Gan dialect, Hakka dialect, and some Shanxi and Shandong dialects. [13] According to the existing materials, the opposition between Zhierzhuang and Zhisanzhang is also preserved in the Jin dialect of the Northern Shaanxi and part of the Central Plains official language.

It is also a long-standing tradition that the pronunciation of Zhishe open-mouth Zhang group in medieval times is the same to that of Zhierzhuang group. Sang Yuhong (2009) found that in the modern Chinese period, there are three kinds of pronunciation expressions in Zhishe open-mouth third-class Zhizhuangzhang group after sorting out the pronunciation of modern Chinese literature. First, Zhikaisanzhi group is same to Zhang group, and opposite to Zhuang group. "List of Ancient and Modern Rhymes", "Basiba", "Mongolian rhyme", and "Xiru Ermu Zi" belong to this category; second, Zhikaisanzhuang group is same to Zhang group, and opposite to Zhi group. "Zhongyuan Yinyun", "Yunlue Yitong", "Yunlue Huitong", "Cheng Tai Yun", and "Five Yuan Fangyin" belong to this category; third, the initial consonant and vowel in Zhikaisanzhi, Zhuang, and Zhang groups are all the same. "Sima Wengong and other rhyme maps" and "Yinyunjicheng" belong to this category. [14] The Northern Shaanxi Jin dialect, including Zhiyan cluster, maintains the opposition between the Zhierzhuang group and Zhisanzhang, and Zhikaisanzhang group has the same pronunciation to Zhuang group, which belongs to the second category. Among several books reflecting this category, the earliest one is "Zhongyuan Yinyun" (1324). When discussing the initial consonants in "Zhongyuan Yinyun", Ning Jifu (1964) pointed out: "The ancient Zhishe open-mouth Zhang group is merged into Zhisiyun and combined with Zhuang group characters". [15] This shows that from the Yuan Dynasty at the latest, Zhikai Sanzhuangzhang group has been merged and is opposite to the Zhi group.

It is the common feature of official language and Jin dialect that Jiangkai Erzhizhuang group and the Dangkai Sanzhuang group is read as round-mouth characters. It is the same in Beijing, Jinan, Taiyuan, Wuhan, Hefei and Yangzhou. Mai Yun (2010) pointed out: "According to the current existing material, the material that first showed the round-mouth pronunciation of Jiangshezhizhao group is probably the "Sisheng dengzi" of the Southern Song Dynasty. As for Dangshezhao group, there is a medial sound ' $h$ ' with the remark of unknown nature in "Mongolia characters rhyme" in Yuan Dynasty." [16] It can be seen from the time sequence that, due to the convergence of Yang Yun and Jiang Yun, the words of Dangkaisanzhuang group were influenced by the words of Jiangkaierzhuang, and they were changed to round-mouth pronunciation.

\section{CONCLUSION}

Li Jianxiao believes that the phonetic pattern of Zhizhuangzhang in the Jin dialect of Northern Shaanxi should have taken shape in the northwestern dialects of the Tang and Five Dynasties. [4] We believe that only two parts of Zhizhuangzhang initial consonant were formed in Tang and Five Dynasties and later the rest pronunciations of Zhikaisanzhang group, Jiangkaierzhizhuang group and the Dangkaisanzhuang groups were gradually formed. The modern pronunciation pattern of Zhizhaozhang groups in Zhiyan cluster is in line with the dialect of "Zhongyuan Yinyun", which was formed at the latest in the Yuan Dynasty. It is precisely because the modern pronunciation pattern of Zhizhuangzhang initial consonants in Zhiyan cluster that has been formed for hundreds of years, and the modern pronunciation pattern of Zhizhuangzhang initial consonants in the surrounding large-scale dialect is the same that the Zhizhuangzhang initial consonants in Zhiyan cluster very stable. Even under today's strong influence of Mandarin, it didn't change obviously. Up to now, the modern pronunciation rules of the old words, immigrant words and new-style words in Zhiyan cluster of Jin dialect are basically the same. The same is true of other northern Shaanxi Jin dialects.

\section{REFERENCES}

[1] Gao Feng. Re-discussion on the geographical distribution and characteristics of the Zhiyan cluster of Jin dialect [J]. Linguistic Researches, 2018 (3). (in Chinese) 
[2] Xiong Zhenghui. Types of ts and ts of dialects in Mandarin dialects [J]. Dialect, 1990 (1). (in Chinese)

[3] Wang Linhui. The phonetic features and transformation of dialects in the Fenhe River Basin [M]. Beijing: China Social Sciences Press, 2003: 64. (in Chinese)

[4] Li Jianxiao. Study on the pronunciation of Jin dialect in northern Shaanxi [D]. Beijing Language and Culture University, 2006. (in Chinese)

[5] Wang Hongjun. The division and combination of Zhizhuangzhang initial consonants in Zhongyuan Yinyun and Its Evolution in Shanxi Dialects[J]. Linguistic Researches, 2007(1). (in Chinese)

[6] Zhang Shuangqing, Xing Xiangdong. The pronunciation type and evolution of round-mouth initial consonants in Zhi system of the Guanzhong dialect $[\mathrm{J}], 2012(2)$. (in Chinese)

[7] Zhang Weijia. The Historical Evolution and Phonetic Conditions of Zhizhuangzhang initial consonants in Guanzhong Dialect[J]. Linguistic Researches, 2012(2). (in Chinese)

[8] Dialect Survey Room of College of Chinese Language and Literature of Northwest Normal University Chinese Department. Overview on Gansu Dialect [G]. Lanzhou: mimeographed manuscript, 1960: 4. (in Chinese)

[9] Zhang Shengyu, Zhang Chengcai. The Division of Chinese Dialects in the Four Provinces of Shaanxi, Gansu, Ningxia and Qinghai[J]. Dialect, 1986(2). (in Chinese)

[10] Zhang Shengyu. The Chinese dialect of the Hexi Corridor. [J]. Dialect, 1993 (4). (in Chinese)

[11] Luo Changpei. The northwestern dialect of the Tang and Five Dynasties [M]. Shanghai: Institute of History and Philology, Academia Sinica, 1933. (in Chinese)

[12] Shao Rongfen. The wrongly written characters and strange articles in Dunhuang folk literature and the dialects in the dynasties of the Tang and Five Dynasties [J]. Chinese, 1963 (3). (in Chinese)

[13] Wan Bo. Research on the historical level of initial consonants of Gan dialect [M]. Beijing: The Commercial Press, 2009: 224-231. (in Chinese)

[14] Sang Yuhong. Several Issues in the Study of Zhizhuangzhang initial consonants in Zhongyuan Yinyun[J]. Study in language and Linguistics, 2009(7). (in Chinese)

[15] Ning Jifu. The twenty-five initial consonants in Zhongyuan Yinyun [J]. Studies of The Chinese Language, 1964 (5). (in Chinese)

[16] Mai Yun. Research on the Zhizhao group from the modern pronunciation and the official Hakka, Xiang, and Gan dialect since the late medieval times [J]. Nankai Linguistics, 2010 (1). (in Chinese) 